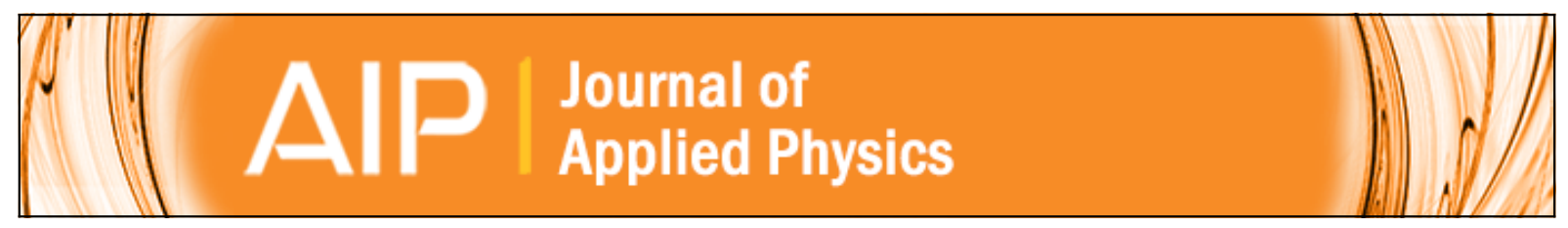

\title{
Laser thermal annealing effects on single crystal gallium phosphide
}

D. Pastor, J. Olea, M. Toledano-Luque, I. Mártil, G. González-Díaz, J. Ibáñez, R. Cuscó, and L. Artús

Citation: Journal of Applied Physics 106, 053510 (2009); doi: 10.1063/1.3187902

View online: http://dx.doi.org/10.1063/1.3187902

View Table of Contents: http://scitation.aip.org/content/aip/journal/jap/106/5?ver=pdfcov

Published by the AIP Publishing

\section{AlP}

\section{Create a profile. \\ Sign up today!}




\title{
Laser thermal annealing effects on single crystal gallium phosphide
}

\author{
D. Pastor, ${ }^{1, a)}$ J. Olea, ${ }^{1}$ M. Toledano-Luque, ${ }^{1}$ I. Mártil, ${ }^{1}$ G. González-Díaz, ${ }^{1}$ J. Ibáñez, ${ }^{2}$ \\ R. Cuscó, ${ }^{2}$ and L. Artús ${ }^{2}$ \\ ${ }^{1}$ Dpto. de Física Aplicada III (Electricidad y Electrónica), Facultad de Ciencias Físicas, Universidad \\ Complutense de Madrid, Madrid E-28040, Spain \\ ${ }^{2}$ Institut Jaume Almera, Consejo Superior de Investigaciones Científicas (CSIC), Lluís Solé i Sabarís s/n, \\ 08028 Barcelona, Spain
}

(Received 24 March 2009; accepted 29 June 2009; published online 4 September 2009)

\begin{abstract}
We have studied the laser thermal annealing (LTA) effects on single crystal GaP. The samples have been analyzed by means of Raman spectroscopy, glancing incidence x-ray diffraction (GIRXD), and transmission electron microscopy (TEM) measurements. After LTA process, the Raman spectra of samples annealed with the highest energy density show a forbidden TO vibrational mode of GaP. This result suggests the formation of crystalline domains with a different orientation in the annealed region regarding the GaP unannealed wafer. This behavior has been corroborated by GIXRD measurements. TEM images show that the LTA produces a defective layer with disoriented crystalline domains in the surface. The depth of this defective layer increases with the energy density of LTA. The lack of crystallinity after LTA processes could be related with the high bond energy value of GaP. (C) 2009 American Institute of Physics. [doi:10.1063/1.3187902]
\end{abstract}

\section{INTRODUCTION}

During past years, many efforts have been carried out to increase the efficiency in solar cells based on single crystal semiconductors. ${ }^{1}$ In 1997, a theoretical paper showed that the introduction in a host semiconductor of a so-called intermediate band (IB) could enhance drastically the efficiency in single junction solar cells. ${ }^{2}$ Since then, further papers describing the properties of such IB have been published. ${ }^{3,4}$ The main feature of an IB material is a new band localized between the conventional valence band (VB) and the conduction band $(\mathrm{CB})$ of the semiconductor. The advantage of this new band is that electrons of energy below the band gap can be promoted from the VB to the CB by means of two subband gap photons absorption of lower energy using the IB as an intermediary.

The IB has been theoretically proposed and experimentally certified in quantum dot (QD) based devices. ${ }^{5,6}$ Currently, intensive research has been realized to improve these QD materials to achieve an optimal efficiency. ${ }^{7}$ The formation of an IB has been shown also in different dilute semiconductors such as GaInNAs, ${ }^{8}$ ZnMnOTe, ${ }^{9}$ and GaNAsP. ${ }^{10}$ However, theoretical calculations point to III-V semiconductors, such as GaAs and GaP, as two of the most interesting materials for the formation of an IB by means of the introduction of deep levels in concentrations above the Mott limit. $^{3,11,12}$ The main drawback of this approach is that the high concentration of impurities required for the IB formation usually exceeds the solid solubility limit. In contrast with most doping methods, ion implantation is a powerful technique that permits the controlled introduction of a high impurity concentration into the bulk semiconductor even above the solubility limit, ${ }^{13}$ allowing to reach the concentration needed for the IB formation. However, the ion implan-

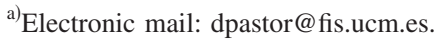

tation damages the crystalline lattice of the host semiconductor, thus thermal annealing treatments are required in order to recover the crystallinity. Given that annealing processes under equilibrium conditions could produce impurity outdiffusion from the semiconductor, nonequilibrium annealing processes are mandatory. In this way, the laser thermal annealing (LTA) process could be a technological key to obtain an IB in bulk semiconductors. ${ }^{10,14-16}$ The effect of the LTA process on III-V semiconductors has been reported previously. ${ }^{17}$ However, as far as we are concerned no detailed study has been carried out on GaP. In this work, we present an optical and structural study of the LTA effects on $\mathrm{GaP}$.

\section{EXPERIMENTAL}

The samples used in this study were undoped liquid encapsulated Czochralsky (LEC) (100) GaP wafers with a thickness of $500 \mu \mathrm{m}$ and a resistivity at room temperature of $\rho=0.2 \Omega \mathrm{cm}$. The samples were annealed at J.P. Sercel Associates, Inc. (New Hampshire, USA) with a KrF excimer laser $(\lambda=248 \mathrm{~nm})$ at energy densities ranging from 0.2 to $1.2 \mathrm{~J} / \mathrm{cm}^{2}$, with a single $20 \mathrm{~ns}$ pulse. For this wavelength we estimate that the annealed region depth in $\mathrm{GaP}$ is of about tens of nanometers. ${ }^{13}$

The Raman spectra were carried out with a Jobin-Yvon T64000 spectrometer equipped with a coupled charge detector cooled with liquid nitrogen. All the spectra were acquired in unpolarized backscattering configuration on a (100) face. The $325 \mathrm{~nm}$ line of a He-Cd laser was used for excitation. For this wavelength, taking into account the corresponding absorption coefficient of $\mathrm{GaP}^{18}$ we estimate that the Raman signal originates from a surface layer thickness of about 10 $\mathrm{nm}$, which is smaller than the depth of the LTA region.

$\mathrm{X}$-ray diffraction measurements were performed to study the crystallinity of the LTA region using an analytical X'Pert PRO MRD diffractometer with $\mathrm{Cu} K_{\alpha}$ radiation in glancing 


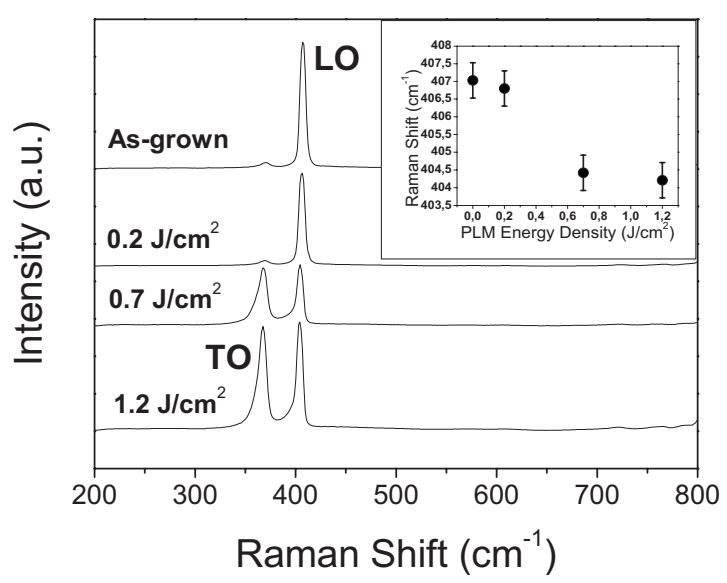

FIG. 1. Room-temperature Raman spectra of LEC-grown GaP layers annealed at different energy densities. Inset: Raman shift versus PLM energy density of the studied samples.

incidence x-ray diffraction (GIXRD) configuration. This technique provides depth-resolved information about the crystal structure by means of the incidence angle of the x-ray source and permits us to probe a depth of tens of nanometers, ${ }^{19}$ about the same thickness order of the LTA layer. Previous to GIRXD measurements, $\theta-2 \theta$ scans were performed to obtain the same orientation sample reference to assess the same sample measurements conditions.

Cross sectional transmission electron microscopy (TEM) images were obtained by a JEOL JEM-2000FX at $200 \mathrm{keV}$. Together with TEM measurements, electron diffraction (ED) patterns with a selected area of diffraction of about $50 \mathrm{~nm}$ were taken.

Stoichiometry control after LTA processes was evaluated by measuring the depth profile of phosphorus atoms in the GaP lattice. These measurements were obtained by time of flight secondary ion mass spectroscopy (TOF-SIMS) performed with a TOF-SIMS IV Gmbh Germany Ion-TOF model. The primary pulsed ion beam consisted of $\mathrm{Bi}^{3+}$ at $45^{\circ}$ incidence with incident energy of $25 \mathrm{keV}$ rastered over a $250 \times 250 \mu \mathrm{m}^{2}$. The $\mathrm{O}_{2}$ gun sputtering ion beam was rastered over a $500 \times 500 \mu \mathrm{m}^{2}$ at $2 \mathrm{keV}$. Depth scales were defined using optical profilometry. Calibration was conducted obtaining $\mathrm{P}$ signal in relation to a nonsaturated reference ion $\left(\mathrm{Ga}^{70}\right)$ in order to correct the secondary ion yield, roughness, and instrumental effects.

\section{RESULTS}

Figure 1 shows unpolarized Raman spectra of $\mathrm{GaP}$ samples annealed at different LTA energy densities $(0.2,0.7$, and $1.2 \mathrm{~J} / \mathrm{cm}^{2}$ ). We also show the spectrum of the unannealed $\mathrm{GaP}$ substrate. According to the selection rules for the zinc-blende structure, only the longitudinal optical (LO) phonon mode is allowed in the backscattering configuration from the (100) face. The Raman spectrum of the unannealed sample is dominated by the peak at $405 \mathrm{~cm}^{-1}$ which is assigned to the LO mode. ${ }^{20}$ Whereas the LO peak of the sample annealed at $0.2 \mathrm{~J} / \mathrm{cm}^{2}$ shows only a slight intensity decrease, the Raman spectra of the samples annealed at energy densities of $0.7 \mathrm{~J} / \mathrm{cm}^{2}$ and higher display a strong transverse optical (TO) peak at $367 \mathrm{~cm}^{-1}$, which is forbidden in this scattering geometry. The observation of a forbidden TO mode in the Raman spectra suggests that the LTA process gives rise to the formation of a GaP layer with disoriented domains, typical of polycrystalline material. A similar behavior has been observed previously in ion implanted InP epilayers after LTA (Ref. 21) and rapid thermal annealing. ${ }^{22}$

Whereas there are no appreciable differences between the LO peak width of the unannealed sample and that of the sample annealed at $0.2 \mathrm{~J} / \mathrm{cm}^{2}$, the LO peak of the sample annealed at $0.7 \mathrm{~J} / \mathrm{cm}^{2}$ shows an asymmetrical broadening to lower frequencies. This increase in the width of the LO peak, together with the presence of a forbidden TO peak, also asymmetrically broadened to lower frequencies indicate a reduction in the crystalline quality, probably induced by defects in the boundary domains of the different orientations.

Since in the spectrum of the sample annealed at $0.2 \mathrm{~J} / \mathrm{cm}^{2}$ only a slight decrease in the intensity of the LO mode is observed, it can be concluded that the LTA process at this energy does not produce a substantial structural change. In contrast, the sample annealed at $0.7 \mathrm{~J} / \mathrm{cm}^{2}$ displays a strong forbidden TO peak (see Fig. 1), which is likely originated from disoriented domains. When the LTA energy density is increased up to $1.2 \mathrm{~J} / \mathrm{cm}^{2}$, the TO and LO vibrational modes still persist. However, in this case both peaks are slightly narrower and more intense, which suggests that a certain improvement of the crystalline quality relative to the sample annealed at $0.7 \mathrm{~J} / \mathrm{cm}^{2}$ has been achieved.

In the inset of Fig. 1 we plot the frequency of the LO mode for the different annealed GaP samples. It is worth noting that the samples annealed with an energy density of 0.7 and $1.2 \mathrm{~J} / \mathrm{cm}^{2}$ exhibit a downward frequency shift of the LO peak of about $3 \mathrm{~cm}^{-1}$. This shift has been consistently observed in different $\mathrm{GaP}$ samples annealed under the same conditions, indicating the presence of strain. Such strain could be related to the formation of crystalline domain boundaries and also with the presence of defects. A disorderinduced reduction in the LO-TO splitting may also explain in part the observed frequency behavior of the LO mode. ${ }^{23}$

The clear structural differences between the sample annealed at the lowest energy density $\left(0.2 \mathrm{~J} / \mathrm{cm}^{2}\right)$ and those annealed at the highest energy densities $\left(0.7\right.$ and $\left.1.2 \mathrm{~J} / \mathrm{cm}^{2}\right)$ suggest that $\mathrm{GaP}$ melting has occurred in the latter. Thus, it can be concluded that the energy density threshold for melting the $\mathrm{GaP}$ surface is within the range of $0.2-0.7 \mathrm{~J} / \mathrm{cm}^{2}$. The formation of differently oriented domains that gives rise to the observation of the forbidden TO mode could be due to a too fast recrystallization rate as temperature quickly falls below the GaP melting point after the LTA annealing. A GaP regrowth layer with disoriented domains could also result from a superficial GaP decomposition since the high density of point defects related to $\mathrm{P}$ vacancies in the shallowest regions could favor the formation of disoriented domains.

In order to further study these structural changes, the crystallinity of the samples was also characterized by means of GIXRD. Figure 2 shows the GIXRD measurements acquired at different glancing angles of the LTA layers at 0.2 , 0.7 , and $1.2 \mathrm{~J} / \mathrm{cm}^{2}$. All these measurements have been car- 

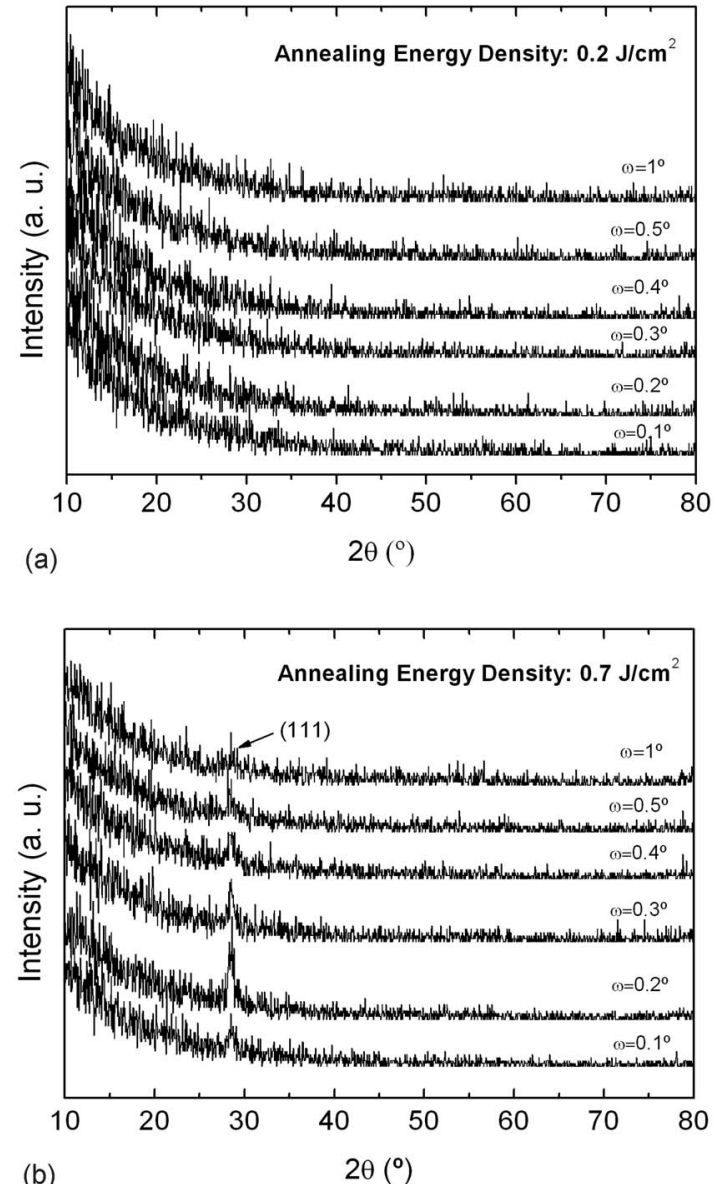

(b)

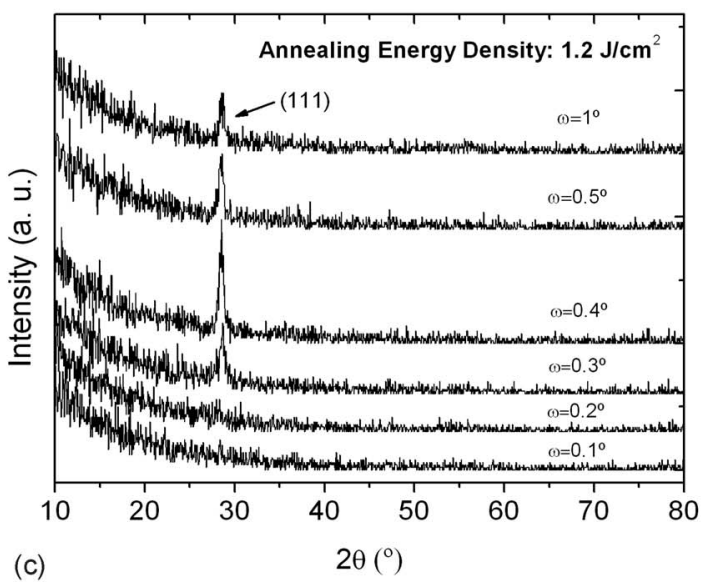

FIG. 2. GIXRD patterns for GaP samples annealed at (a) $0.2 \mathrm{~J} / \mathrm{cm}^{2}$, (b) $0.7 \mathrm{~J} / \mathrm{cm}^{2}$, and (c) $1.2 \mathrm{~J} / \mathrm{cm}^{2}$. These measurements were taken at glancing angles of $\omega=0.1^{\circ}, 0.2^{\circ}, 0.3^{\circ}, 0.4^{\circ}, 0.5^{\circ}$, and $1^{\circ}$.

ried out at the same sample orientation. No peaks have been observed in the sample annealed with the lowest density energy $\left(0.2 \mathrm{~J} / \mathrm{cm}^{2}\right)$ neither in unannealed sample (not shown here). In fact, there is not any appreciable difference in the GIRXD patterns between the unannealed sample and the sample annealed at $0.2 \mathrm{~J} / \mathrm{cm}^{2}$. This result shows that the annealing at $0.2 \mathrm{~J} / \mathrm{cm}^{2}$ does not produce any significant effect in the $\mathrm{GaP}$ lattice structure.

However, the GIXRD patterns of the samples annealed at 0.7 and $1.2 \mathrm{~J} / \mathrm{cm}^{2}$ present a new peak attributed to the (111) reflection of GaP. This peak could be due to the ap- pearance of different orientated domains from the GaP unannealed region. Figures 2(b) and 2(c) show that the intensity of the peak increases with increasing glancing angle and then decreases for higher angles. However, whereas the sample annealed at $0.7 \mathrm{~J} / \mathrm{cm}^{2}$ has the maximum at $\omega=0.2^{\circ}$, the sample annealed at $1.2 \mathrm{~J} / \mathrm{cm}^{2}$ has the highest peak intensity at $\omega=0.4^{\circ}$. This behavior can be explained in terms of an increase in the annealed region as the annealing density energy is increased (as we will see later). For these angles, the sample annealed at the highest energy density shows also the highest intensity, corresponding in part to a higher diffracted volume of the annealed region.

Moreover, the narrower width of this peak and also in part, the highest intensity in the sample annealed at the higher energy density $\left(1.2 \mathrm{~J} / \mathrm{cm}^{2}\right)$ with regard to the sample annealed at $0.7 \mathrm{~J} / \mathrm{cm}^{2}$, could indicate an increase in the size of the different disorientated crystalline domains, and thus, a decrease in effective area of defects. All these results are in full agreement with the results obtained by Raman spectroscopy.

Figure 3 shows the cross sectional TEM images of the LTA samples. In Fig. 3(a), as result of the LTA annealing at $0.2 \mathrm{~J} / \mathrm{cm}^{2}$, a superficial defective layer of about $10 \mathrm{~nm}$ can be appreciated on the top of the monocrystalline GaP substrate. This superficial defective layer explains the slight differences regarding the unannealed sample observed in the Raman and GIRXD measurements.

As LTA energy density is increased, the defective layer depth increases. On the top of sample annealed at $0.7 \mathrm{~J} / \mathrm{cm}^{2}$ [Fig. 3(b)] a defective layer about $20 \mathrm{~nm}$ thick with different orientated domains can be observed. The boundary domains are clearly differentiated and cross the defective layer from the unaltered substrate to the surface. This suggests some preferential orientations of the domains. When the energy is increased up to $1.2 \mathrm{~J} / \mathrm{cm}^{2}$ [Fig. 3(c)], the depth of the defective layer is increased up to a depth of $50 \mathrm{~nm}$. The disoriented domains have a larger size and show in some areas a columnar structure which is consistent with a preferential orientation. This preferential domain orientation is supported by the GIRXD measurements where only one GaP diffraction plane is observed in the diffraction patterns. The increase in the domain size as LTA energy increases suggests the possibility to obtain higher domains increasing more this annealing energy.

The differences in the depth of the defective layer are also in agreement with the differences observed in the intensity of the diffraction peak obtained in the GIXRD patterns. The diffraction volume of the defective layer increases with increasing LTA energy density so we obtain higher intensity peaks for the samples annealed with the highest energy density.

Figure 4 shows the ED patterns for the same samples of the TEM images. The ED pattern of the sample annealed at $0.2 \mathrm{~J} / \mathrm{cm}^{2}$ [Fig. 4(a)] does not differ from the ED pattern of the unannealed GaP sample (not shown here), in clear agreement with Raman and GIXRD measurements. Whereas the ED patterns of the samples LTA annealed at 0.7 and $1.2 \mathrm{~J} / \mathrm{cm}^{2}$ [Figs. 4(b) and 4(c)] in the unannealed region showed the typical GaP diffraction pattern (the same as in 


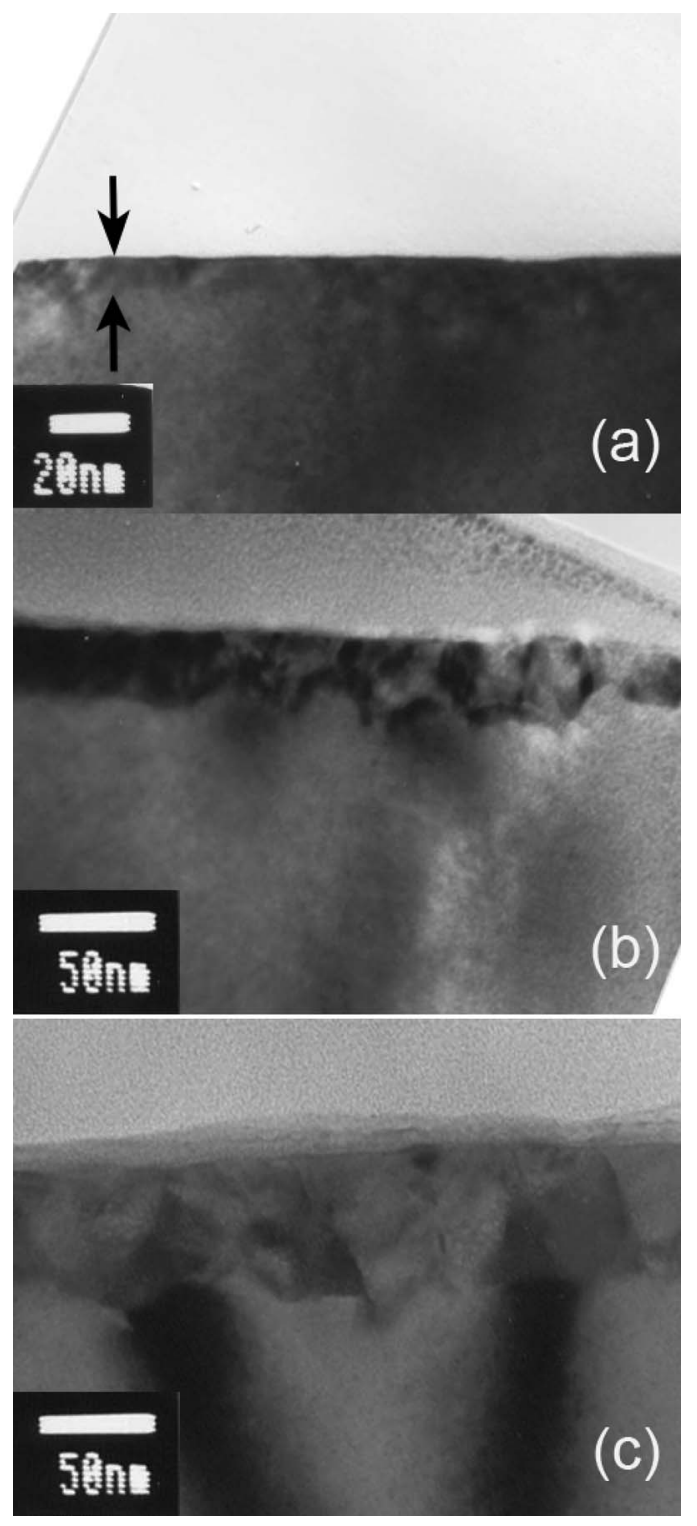

FIG. 3. Cross sectional TEM images of the samples annealed at (a) $0.2 \mathrm{~J} / \mathrm{cm}^{2}$, (b) $0.7 \mathrm{~J} / \mathrm{cm}^{2}$, and (c) $1.2 \mathrm{~J} / \mathrm{cm}^{2}$.

the $0.2 \mathrm{~J} / \mathrm{cm}^{2}$ sample), the defective regions present an unusual feature. Between the main bright spots, which correspond to the $[100]$ axis of the GaP, additional spots located only in some preferential directions become visible. This effect indicates a highly defective lattice. ${ }^{24}$ On the other hand, these additional spots cannot be distinguished from the [100] dots for the samples annealed at the highest energy density. However, other spots of weak intensity can be observed out of the preferential directions that point out to a disorientation of the domains with respect to the substrate. Although the size of disoriented domains increases at the highest annealing energy density, this takes place with a high defect concentration.

LTA processes have been extensively used on ion implanted III-V compound semiconductors to recrystallize ion implanted damaged regions. ${ }^{25}$ In fact, using the LTA method, ion implanted amorphized layers of different As-based semiconductors such as GaAs, ${ }^{25} \mathrm{GaN}_{x} \mathrm{As}_{1-x},{ }^{26}$ and $\mathrm{Ga}_{1-x} \mathrm{Mn}_{x} \mathrm{As}$ (Ref. 27) could be recrystallized into single crystal. In our

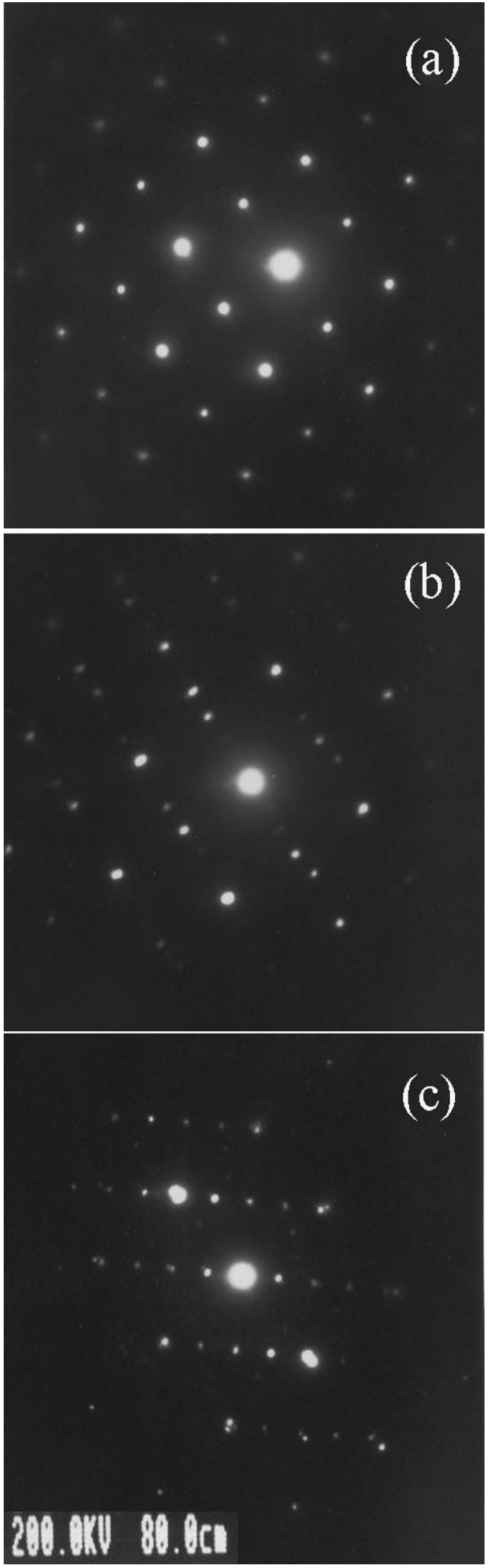

FIG. 4. ED images of the samples annealed at (a) $0.2 \mathrm{~J} / \mathrm{cm}^{2}$, (b) $0.7 \mathrm{~J} / \mathrm{cm}^{2}$, and (c) $1.2 \mathrm{~J} / \mathrm{cm}^{2}$.

case, as Figs. 2-4 point out, no single crystal has been achieved after LTA annealings, even without ion implantation treatments. This is a striking fact even if we consider that LTA energies used in our experiments are of the order or 


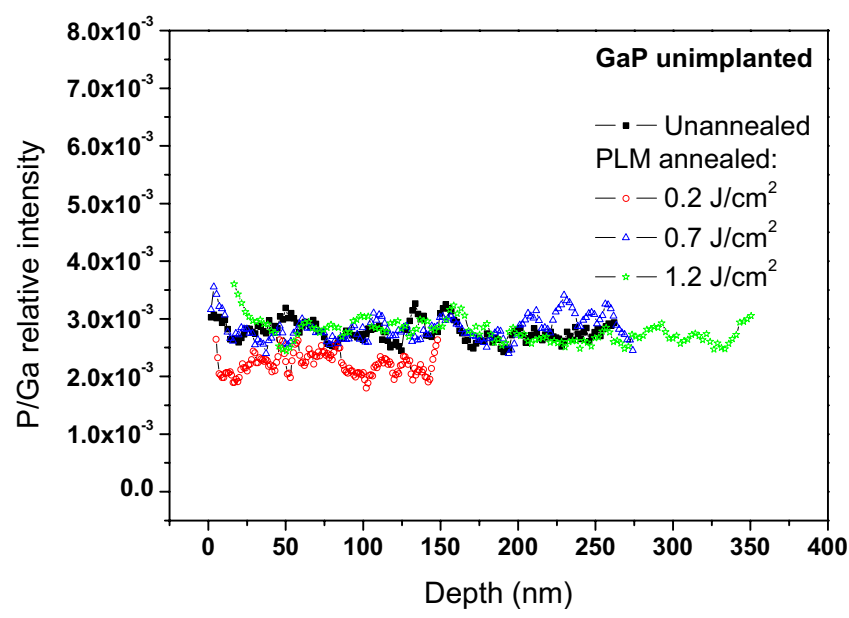

FIG. 5. (Color online) Phosphorus related Ga signal as a function of sample depth for unannealed sample and LTA treated samples at 0.2, 0.7, and $1.2 \mathrm{~J} / \mathrm{cm}^{2}$.

higher than those values reported for the As-based semiconductors $\left(0.2-0.4 \mathrm{~J} / \mathrm{cm}^{2}\right),{ }^{26,27}$ where single crystal regrowth was successfully achieved.

A cause of concern is that the annealing treatments at high temperature realized in III-V semiconductors can produce the evaporation of the most volatile element of the alloy. When equilibrium annealing treatments are used, this drawback is usually overcome performing the thermal treatments in an overpressure atmosphere of this more volatile component or using a capping. In GaP, a loss of $\mathrm{P}$ would break the stoichiometry and could explain the polycrystalline behavior after LTA treatment. As an attempt to elucidate the origin of this lack of crystallinity, we have analyzed the $\mathrm{P}$ concentration depth profile by means of TOF-SIMS measurements after the different LTA treatments performed. Figure 5 displays the depth profile of the phosphorous intensity signal related to $\mathrm{Ga}$ of samples LTA annealed at 0.2, 0.7, and $1.2 \mathrm{~J} / \mathrm{cm}^{2}$. In these profiles, no phosphorous loss is detected after LTA processes for the different energy densities. This seems to rule out the generation of P vacancies after LTA as the cause of polycrystallinity.

On the other hand, it is worth noting that the high density photoexcitation of bonding electrons generated by the LTA produces structure instability in $\mathrm{GaP}$ single crystals and atomic rearrangement even with a few hundreds of femtoseconds. ${ }^{28}$ In this way, the most probable mechanism underlying the lack of crystallinity of our GaP LTA treated samples could be related with different values of the singlebond energy of $\mathrm{GaP}$ compared to GaAs. In fact, this value is $20 \%$ higher in GaP compared to GaAs. ${ }^{29,30}$ As a consequence, recrystallization processes could be more difficult to take place in $\mathrm{GaP}$ in comparison with $\mathrm{GaAs}$. This agrees with the fact that the efficiency of recombination enhanced defect creation increases with the increase in bandgap energy. Defect generation after LTA annealings for GaP at energy densities below a threshold has been reported previously, ${ }^{31}$ suggesting that the defects could be localized in a superficial layer of $30-50 \mathrm{~nm}$. That is completely in agreement with our results where added to defects, a superficial polycrystalline layer is originated after LTA treatments.

\section{CONCLUSIONS}

Summarizing, the observation of forbidden vibrational Raman modes in LTA samples indicates polycrystalline surface layer formation. These additional forbidden peaks are not clearly visible up to LTA energy density of $0.7 \mathrm{~J} / \mathrm{cm}^{2}$, suggesting a threshold energy density beyond which $\mathrm{GaP}$ melting occurs. TEM images and GIRXD measurements, in agreement with the Raman spectroscopy results, show disorientated crystalline domains after annealing at the highest energy densities suggesting highly differentiated domains. Since the TOF-SIMS profiles do not show phosphorus loss after LTA treatments, we ruled out the compositional changes as the cause of the lack of crystallinity present in our samples after the LTA treatments. We tentatively suggest that the lack of crystallinity after LTA is in relation with the energy bond value. All these effects constitute a handicap for achieving IB formation in $\mathrm{GaP}$ by means of ion implantation and subsequent LTA processing.

\section{ACKNOWLEDGMENTS}

The authors would like to acknowledge the CAI de Difracción de Rayos X and CAI de Microscopía of the Universidad Complutense de Madrid for GIXRD measurements and TEM measurements, respectively. This work was made possible because of the FPI program (Grant No. BES-20057063) and the Spanish Ministry of Education and Science under Contract No. MAT2007-63617. This work was partially supported by the Project NUMANCIA (Grant No. S-0505/ENE/000310) funded by the Comunidad de Madrid and by the Project GENESIS-FV (Grant No. CSD200600004) funded by the Spanish Consolider National Program.

${ }^{1}$ A. Luque and A. Martí, Prog. Photovoltaics 9, 73 (2001).

${ }^{2}$ A. Luque and A. Martí, Phys. Rev. Lett. 78, 5014 (1997).

${ }^{3}$ A. Luque, A. Marti, E. Antolín, and C. Tablero, Physica B 382, 320 (2006).

${ }^{4}$ A. Martí, L. Cuadra, N. Lopez, and A. Luque, Semiconductors 38, 946 (2004).

${ }^{5}$ L. Cuadra, A. Martí, and A. Luque, IEEE Trans. Electron Devices 51, 1002 (2004)

${ }^{6}$ A. Martí, E. Antolín, C. R. Stanley, C. D. Farmer, N. López, P. Díaz, E. Cánovas, P. G. Linares, and A. Luque, Phys. Rev. Lett. 97, 247701 (2006). ${ }^{7}$ A. Luque, A. Martí, N. López, E. Antolín, E. Cánovas, C. Stanley, C. Farmer, and L. J. Caballero, L. Cuadra, and J. L. Balenzategui, Appl. Phys. Lett. 87, 083505 (2005).

${ }^{8}$ W. Shan, W. Walukiewicz, J. W. Ager III, E. E. Haller, J. F. Geisz, B. J. Friedman, J. M. Olson, and S. R. Kurtz, Phys. Rev. Lett. 82, 1221 (1999).

${ }^{9}$ K. M. Yu, W. Walukiewicz, J. Wu, W. Shan, J. W. Beeman, M. A. Scarpulla, O. D. Dubon, and P. Becla, Phys. Rev. Lett. 91, 246403 (2003).

${ }^{10}$ K. M. Yu, W. Walukiewicz, J. W. Ager III, D. Bour, R. Farshchi, O. D. Doubon, S. X. Li, D. Sharp, and E. E. Haller, Appl. Phys. Lett. 88, 092110 (2006).

${ }^{11}$ C. Tablero and P. Wahnón, Appl. Phys. Lett. 82, 151 (2003).

${ }^{12}$ C. Tablero, Phys. Rev. B 72, 035213 (2005).

${ }^{13}$ J. Olea, M. Toledano-Luque, D. Pastor, G. González-Díaz, and I. Mártil, J. Appl. Phys. 104, 016105 (2008).

${ }^{14}$ K. M. Yu, W. Walukiewicz, J. Wu, W. Shan, J. W. Beeman, M. A. Scarpulla, O. D. Dubon, and M. C. Ridgway, Appl. Phys. Lett. 83, 2844 (2003).

${ }^{15}$ E. Antolín, A. Martí, J. Olea, D. Pastor, G. González-Díaz, I. Mártil, and A. Luque, Appl. Phys. Lett. 94, 042115 (2009).

${ }^{16}$ J. Olea, G. González-Díaz, D. Pastor, and I. Mártil, J. Phys. D: Appl. Phys. 42, 085110 (2009).

${ }^{17}$ K. M. Yu, Semicond. Sci. Technol. 17, 785 (2002)

${ }^{18}$ D. E. Aspnes and A. A. Studna, Phys. Rev. B 27, 985 (1983). 
${ }^{19}$ E. D. Specht, C. J. Sparks, and C. J. Mc Hargue, Appl. Phys. Lett. 60, 2216 (1992).

${ }^{20}$ M. V. Hobden and J. P. Rusell, Phys. Lett. 13, 39 (1964).

${ }^{21}$ G. Vitali, C. Pizzuto, G. Zollo, D. Karpuzov, M. Kalitzova, P. van der Heide, G. Scamarcio, V. Spagnolo, L. Chiavarone, and D. Manno, Phys. Rev. B 59, 2986 (1999).

${ }^{22}$ L. Artús, R. Cuscó, J. Ibáñez, J. M. Martin, and G. González-Díaz, J. Appl. Phys. 82, 3736 (1997).

${ }^{23}$ G. Burns, F. H. Dacol, C. R. Wie, E. Burstein, and M. Cardona, Solid State Commun. 62, 449 (1987).

${ }^{24}$ D. B. Williams and B. C. Carter, Transmission Electron Microscopy. II, Diffraction (Plenum, New York, 1996).

${ }^{25}$ C. W. White and P. S. Peeercy, Laser and Electron Beam Processing of
Materials (Academic, New York, 1980).

${ }^{26}$ K. M. Yu, W. Walukiewicz, O. D. Dubon, J. Jasinski, Z. Liliental-Weber, J. Wu, J. W. Beeman, M. R. Pillai, and M. J. Aziz, J. Appl. Phys. 94, 1043 (2003).

${ }^{27}$ M. A. Scarpulla, O. D. Dubon, K. M. Yu, O. Monteiro, M. R. Pillai, M. J. Aziz, and M. G. Ridgway, Appl. Phys. Lett. 82, 1251 (2003).

${ }^{28}$ G. Vitali, L. Palumbo, M. Rossi, G. Zollo, C. Pizzuto, L. Di Gaspare, and F. Evangelisti, Phys. Rev. B 53, 4757 (1996).

${ }^{29}$ P. Manca, J. Phys. Chem. Solids 20, 268 (1961).

${ }^{30}$ R. R. Reddy, S. Anjaneyulu, and C. L. N. Sharma, J. Phys. Chem. Solids 54, 635 (1993).

${ }^{31}$ V. I. Emel'yanov and P. K. Kashkarov, Appl. Phys. A: Solids Surf. 55, 161 (1992). 